\title{
Geant4-GATE Simulation of a Large Plastic Scintillator for Muon Radiography
}

\author{
P. Aguiar, E. Casarejos, J. Silva-Rodríguez, J. A. Vilan, and A. Iglesias
}

\begin{abstract}
Envisaging the possibility of using large-area plastic scintillator slabs as robust detectors for high spatial resolution muon radiography, and prior to prototype development, we study expected basic performance by Monte Carlo simulation. We present preliminary results for a scalable square footprint detector unit of $\sim 1 \mathrm{~m}^{2}$, defining a representative simulation model volume of $50 \mathrm{~cm} \times 50 \mathrm{~cm}$, with reflective surfaces and a light readout by direct coupling of 4 small PMTs (in a square arrangement) at one face of the scintillator slab. Light detection efficiency is calculated for several light collection configurations, considering different values of surface roughness, reflectivity, optical coupling index and scintillator thickness. Values maximizing photon detection have been identified. The light response function of $2.5-3.5 \mathrm{~cm}$ diameter PMTs for the proposed configuration has been determined. A detector intrinsic spatial resolution of the order of $1 \mathrm{~cm}$ is estimated for muon interactions at the center region of the detector module, using a simple centroid positioning algorithm (Anger logic).
\end{abstract}

Index Terms-Cosmic rays, GATE, Geant4, homeland security, muon radiography, muons.

\section{INTRODUCTION}

$\mathbf{M}$ UON Radiography has been used (since 1955 [1]) for passive inspection of large- volume objects in a number of applications in volcanology, civil engineering, vessel industry and security. Conventionally, a combination of plastic scintillator detector planes (square or strip segmented) has been used to track muon trails in muon radiography [2]-[5]. Plastic scintillators require minimal maintenance, and a limited number of readout channels, and are relatively insensitive to ambient conditions. Segmented scintillator strips may attain good results, depending on the number of detection planes and their segmentation size. But their spatial resolution is limited by mechanical segmentation and the need of a high number of photo-detectors (readout channels). Detector spatial resolution is a crucial parameter for the quality of muon tracking and imaging results. Recently, high-resolution detectors (drift tubes or micro-pattern gas detectors, achieving a spatial resolution of

Manuscript received July 29, 2013; revised April 15, 2014; accepted April 23, 2015. Date of current version June 12, 2015.

P. Aguiar and J. Silva-Rodríguez are with Fundacion Ramón Domínguez, Complexo Hospitalario Universitario de Santiago de Compostela, 15706 Santiago de Compostela, Spain (e-mail: pablo.aguiar.fernandez@sergas.es; jesus. silva@gmx.es).

E. Casarejos, J. A. Vilan, and A. Iglesias are with Grupo CIMA, Dpto de Enxeñería Mecánica, Univ. de Vigo, 36310 Vigo, Spain (e-mail: e.casarejos@uvigo.es; jvilan@uvigo.es; alfredo.iglesias@uvigo.es;).

Color versions of one or more of the figures in this paper are available online at http://ieeexplore.ieee.org.

Digital Object Identifier 10.1109/TNS.2015.2431297 about 50 micron) have been proposed for muon tomography in security applications [6]-[8]. But the operation of large-area gas detector systems in the real world of industrial applications, where the requirements of robustness and maintenance rule out sophisticated solutions, has still to be demonstrated. Several muon detection systems using 3-5 cm section strip-segmented plastic scintillators, are been developed for geophysical applications [9], cargo inspection [10] or concreted nuclear waste inspection [11], giving $0.1 \mathrm{rad}$ angular resolution, $5 \mathrm{~cm}$ or $1 \mathrm{~cm}$ voxel size tomographic images respectively. In this work we envisage the possibility of using large-area unsegmented plastic scintillator slabs as solid and robust detectors to achieve comparable spatial resolution values. Prior to any prototype development, we study the expected basic performance by Monte Carlo simulation with GATE (Geant4 Application for Emission Tomography: a simulation toolkit for PET and SPECT). This tool has been shown to produce reliable MC simulation results including optical transport, and enables integration of low-level results into complex detection structures for future system $R \& D$ [12]. A basic detector module design is proposed based on the use of a large-area ( $\sim 1 \mathrm{~m}$ size) square scintillator slab, and conventional components: small photomultiplier tubes (PMTs), reflector foil and optical coupling gel. A "gammacameralike" light readout scheme is considered, where an uniformly (sparse) distributed arrangement of small PMTs is coupled to the scintillator face.

The aim of this study is to evaluate the feasibility of the proposed detector module for cosmic muon detection in terms of intrinsic spatial resolution, and its dependence on main detector parameters (such as scintillator thickness and surface finishing or PMT active area size and spacing), as a basic input for a first estimation of muon tracking system performance (and cost) depending on the number of detection planes and their relative distances. A possible set-up based on large square scintillator slabs with such a configuration is shown in Fig. 1.

\section{Materials AND MethodS}

\section{A. Description of the Detector Module}

1) Large-Area Scintillator Slab: A detector module equivalent to a $100 \mathrm{~cm} \times 100 \mathrm{~cm}$ plastic scintillator slab is proposed. The scintillator's physical properties are those of a general purpose BC-408 scintillator [13]. This scintillator has a light yield of 10,000 photons $/ \mathrm{MeV}$ with maximum emission at $423 \mathrm{~nm}$, a bulk attenuation length of $380 \mathrm{~cm}$ and a refractive index of 1.58. The slab thickness reference value is $1.27 \mathrm{~cm}$ but larger values are considered. The selection of scintillator surface finish 


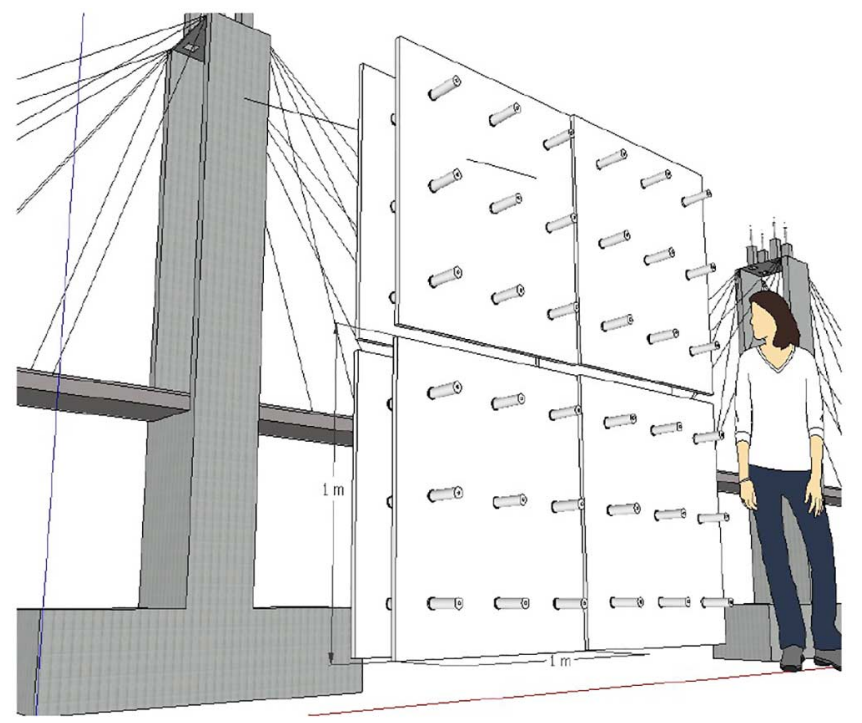

Fig. 1. Artist's view of a possible muon radiography system set-up for largevolume inspection. A scalable system based on large scintillator slabs covered by a square footprint distribution of photodetectors.

is based on a previous work showing the effect of high reflectivity and surface roughness [14]. The $100 \times 100 \mathrm{~cm}^{2}$ faces are rough surfaces, covered with highly reflective foil (3M Vikuiti ESR [15] with a reflectivity coef. of $\mathrm{RC}=0.98$ ). Scintillator slab edges are defined as rough surfaces and covered with black coating $(\mathrm{RC}=0.075)$ to avoid back reflections at the scintillator border. A schema showing the different components of the detector setup is shown in Fig. 2.

2) Light Readout Configuration: A conventional light detection solution based on small PMTs coupled to the scintillator surface, with a light collection window of about 1" diameter readout area, is considered for limited-cost, compact, rough, and stable low-intensity light detection. This direct readout setup on one scintillator slab face is easily feasible, and prevents large dead space between detector modules. As a simple arrangement of the photo-detectors for uniform coverage of the scintillator surface, a square footprint distribution of photodetectors is considered, defining a minimum spacing of $25 \mathrm{~cm}$ (see Fig. 3).

3) Geant4-GATE Simulation Model: A minimum detector model volume of $50 \mathrm{~cm} \times 50 \mathrm{~cm} \times 1.27 \mathrm{~cm}$ is considered for simulation. The coordinate system is defined at the middle of the simulated volume. Fig. 2 shows a sketch of the simulated detector model geometry. The dimensions and optical parameter values of the detector model elements considered in the simulation are summarized in Table I. The reflective foil is modeled as an additional volume at a distance (air gap) of $0.1 \mathrm{~mm}$ from the scintillator surface. Different surface roughness values (standard deviation of surface micro-facet, sigma $=0$ to 9 ) and foil reflectivity values ( $\mathrm{RC}=0$ to 1$)$ are considered to investigate the behavior of a nonspecular reflector. To simulate realistic conditions of the PMT window coupling a transparent filling material with an appropriate refractive index to maximize light collection at the PMT photocathode is considered. A thin volume $(0.2 \mathrm{~mm}$ thick) of optical coupling material is defined. Different index values are considered $\left(\mathrm{n}_{-} \mathrm{OC}=\right.$ $1.50,1.59,1.62,1.70)$.

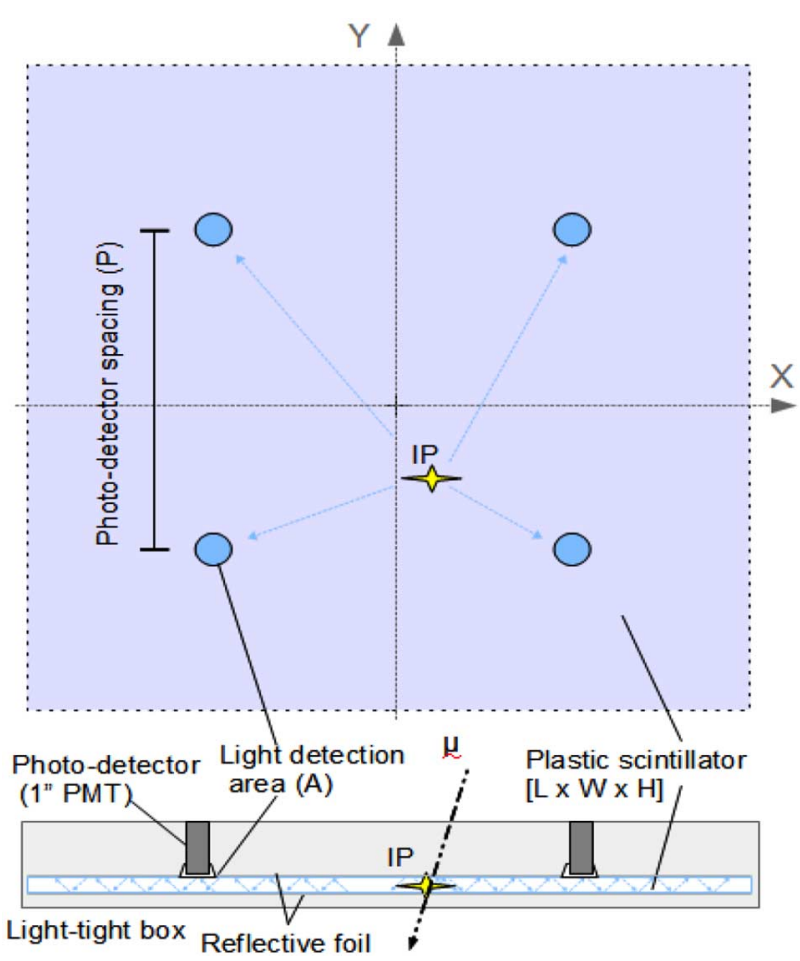

Fig. 2. Schema of the simulated scintillator detector volume. Reference values of detector volume size $\mathrm{L} \times \mathrm{W}=50 \mathrm{~cm} \times 50 \mathrm{~cm}$, scintillator thickness $\mathrm{H}=$ $1.27 \mathrm{~cm}$, photodetector (PD) light collection area diameter $\mathrm{A}=2.5 \mathrm{~cm}$ and $\mathrm{PD}$ spacing $\mathrm{P}=25 \mathrm{~cm}$ are considered for the simulation. The vertical cut shows the different components of the detector simulation model for the proposed light readout configuration.

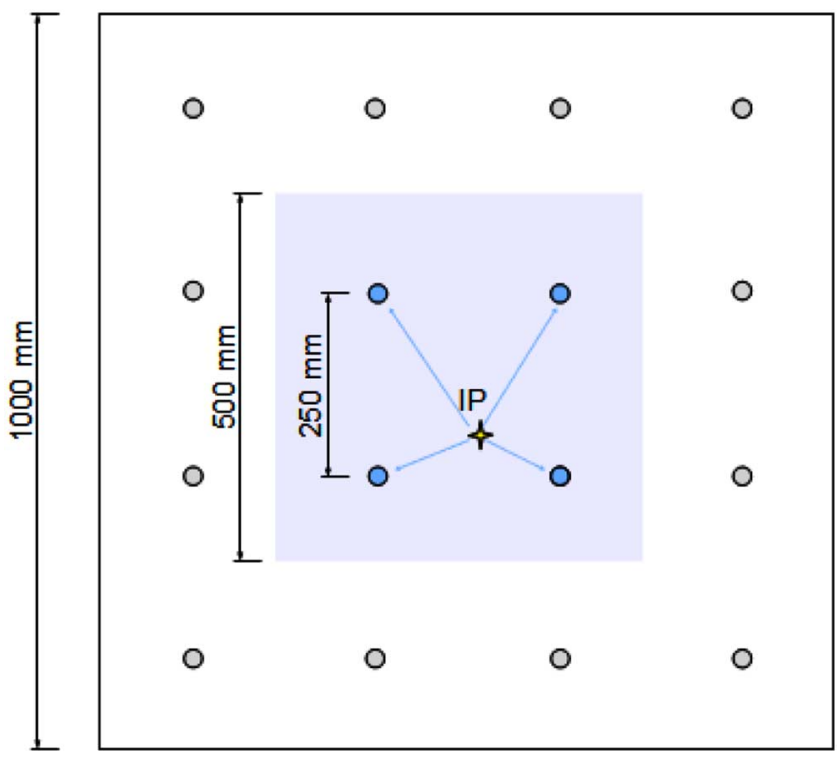

Fig. 3. Layout scheme of a possible sparse distribution of photodetectors $(4 \times 4$ square footprint) covering a large square scintillator slab face $\left(\sim 1 \mathrm{~m}^{2}\right)$. A minimum detector model volume corresponding to a $50 \mathrm{~cm} \times 50 \mathrm{~cm}$ detector central region (shadowed region) is considered for this $\mathrm{MC}$ simulation study.

Muons with a momentum of $4 \mathrm{GeV}$ are defined to simulate cosmic muons (cosmic muons have about $4 \mathrm{GeV}$ mean energy at the ground) passing perpendicularly to the scintillator slab at 
TABLE I

Elements of the Simulated Detector Model. Dimensions and Optical Parameter Values (and Ranges) Considered in the Simulation

\begin{tabular}{|c|c|c|}
\hline Detector element & Dimensions & Optical parameters \\
\hline $\begin{array}{l}\text { Scintillator slab } \\
\text { (gral purpose } \\
\text { scint. BC-408) }\end{array}$ & $\begin{array}{l}\text { Width x Length: } \\
100 \times 100 \mathrm{~cm} 2 \\
\text { Thickness: } \\
\mathrm{H} \geq 1.27 \mathrm{~cm} \\
(1.27-4.0 \mathrm{~cm})\end{array}$ & $\begin{array}{l}\text { Light yield: } \\
\text { 10,000 photons/MeV } \\
\text { Light attenuation length: } \\
380 \mathrm{~cm} \\
\text { Scint. refraction index: } \\
\text { n_scint }=1.58\end{array}$ \\
\hline $\begin{array}{l}\text { Scintillator } \\
\text { surface finish }\end{array}$ & $\begin{array}{l}\text { Polished slab } \\
\text { faces (W x L) } \\
\text { Black painted slab } \\
\text { borders }\end{array}$ & $\begin{array}{l}\text { Roughness: } \\
\text { sigma }=3.0(0-9) \\
\text { Black paint reflectivity: } \\
\text { RC_black }=0.075\end{array}$ \\
\hline Reflector foil & $\begin{array}{l}\text { Scintillator slab } \\
\text { Width x Length }\end{array}$ & $\begin{array}{l}\text { Reflector reflectivity: } \\
\text { RC_reflec }=0.98(0-1)\end{array}$ \\
\hline Photodetector & $\begin{array}{l}\text { Photocathode } \\
\text { diameter: } 2.5 \mathrm{~cm} \\
\text { PD window } \\
\text { thickness: } 1.0 \mathrm{~mm} \\
\text { Optical coupling } \\
\text { thickness: } 0.2 \mathrm{~mm}\end{array}$ & $\begin{array}{l}\text { PD efficiency: } \\
\text { QE }=25 \% \\
\text { PD window index: } \\
\text { n_PD }=1.55 \\
\text { Optical coupling index: } \\
\text { n_OC } \geq 1.5(1.5-1.7)\end{array}$ \\
\hline
\end{tabular}

a given position. A number of photons are created in the scintillator (proportional to the energy deposit of the muon) along the track of the muon.

\section{B. Basic Performance Simulation}

1) Light Collection Efficiency: Light collection efficiency is the critical aspect determining light signal statistics and thereby the uncertainty of muon interaction point estimation. Each muon creates about 30,000 photons (in a $1.27 \mathrm{~cm}$-thick plastic scintillator) propagating inside the scintillator acting as a light guide. Each photon is reflected many times (a long path) at the scintillator surface until it hits one of the PMTs photocathode (with a relatively small sensitive area) and is converted into a photoelectron (with a $25 \%$ probability, for a typical PMT quantum efficiency).

The expected number of photoelectrons per PMT (for a given photodetector active area) is the first interesting property of the setup to be estimated. The effect of surface roughness, reflectivity or scintillator thickness on photon propagation and light collection efficiency is studied, in terms of average number of photoelectrons obtained from multiple simulation runs (with $10 \%$ uncertainty), using a detailed optical transport model defined in the MC simulation with toolkit GATE [12]. The muon interaction point is defined as beeing in the middle of the simulated volume (origin of the coordinate system).

2) Photodetector Light Response Function: The number of photons arriving at a PMT depends on its distance to the muon interaction point. The spatial distribution at the readout face of these optical photons is described by the light spread function (LSF). The dependency gradient and the statistical fluctuations of the number of photons determine the intrinsic spatial resolution of the PMT readout. It is a key issue to analyze this dependency, defined as the photodetector light response function (LRF), for a given light readout configuration (scintillator thickness, roughness, reflectivity, optical coupling). LRF is analyzed

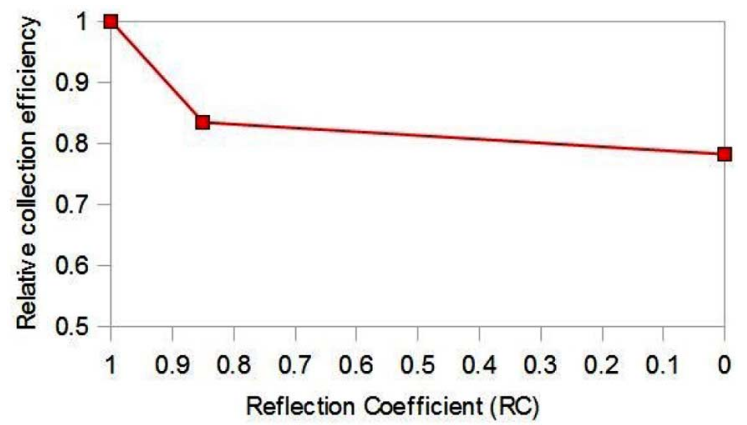

Fig. 4. Light collection dependence on reflector foil reflectivity, $\mathrm{RC}=0$ to 1. Scintillator thickness $\mathrm{H}=1.27 \mathrm{~cm}$; surface roughness sigma $=0$; optical coupling index $\mathrm{n}_{-} \mathrm{OC}=1.7$.

in terms of average photodetector light response function, by computing the number of photoelectrons obtained from multiple simulation runs for several distances of interaction point to PMT. Different muon interaction points are defined along the $X$ axis and $\mathrm{Y}$ axis ( $\mathrm{Y}$ or $\mathrm{X}=0 \mathrm{~cm}, 2.5 \mathrm{~cm}, 5 \mathrm{~cm}, 10 \mathrm{~cm}, 15 \mathrm{~cm}$ and $20 \mathrm{~cm}$ ). To study the LRF shape dependency with the photodetector active area, two different PMT photocathode diameters have been considered in simulations: a $2.5 \mathrm{~cm}$ and a $3.5 \mathrm{~cm}$ diameter active area.

3) Spatial Resolution: One of the main issues of this work is to have an estimation of the muon detection spatial resolution that can be achieved with the proposed readout configuration. The intrinsic spatial resolution of the detector is determined by the uncertainty in the positioning of the muon interaction points, defined as the width of the estimated interaction Point Spread Function (PSF) distribution. Spatial coordinates $(\mathrm{x}, \mathrm{y})$ of individual muon interaction events can be determined from PMT signal values (integrated light pulse charge generated by the PMT) and their relative positions (2D LSF spatial sampling), using a simple centroid positioning algorithm (Anger logic [16]). This algorithm calculates a weighted sum of the individual PMT signals receiving light and normalizes it with the total signal obtained from the PMTs. The weights for the PMT signals depend on the PMT position within the $2 \times 2$ array. The positioning dispersion will be determined by the statistical variations in the light reaching the PMTs. As a simple arrangement of $2 \times 2$ PMTs is considered for uniform coverage of the minimum detector volume area defined in the simulation $(50 \mathrm{~cm} \times 50 \mathrm{~cm}$ ), a minimum set of only four PMT signal values is used in the positioning algorithm. See Fig. 2.

\section{RESUlTS AND Discussion}

1) Light Collection Efficiency: Reflective foil reflectivity: To study the influence of the reflector foil on light transport, the reflectivity parameter is varied over a range of values $\mathrm{RC}=0$ to 1 . The use of a high reflectivity foil, with $\mathrm{RC}=0.98$, shows a significant impact on photon transport (see Fig. 4), giving a 20\% increase in light collection compared to a configuration without reflective foil (only scintillator-to-air reflections).

Optical coupling: To study the influence of the PMT optical coupling on light detection, the refractive index is varied over a 


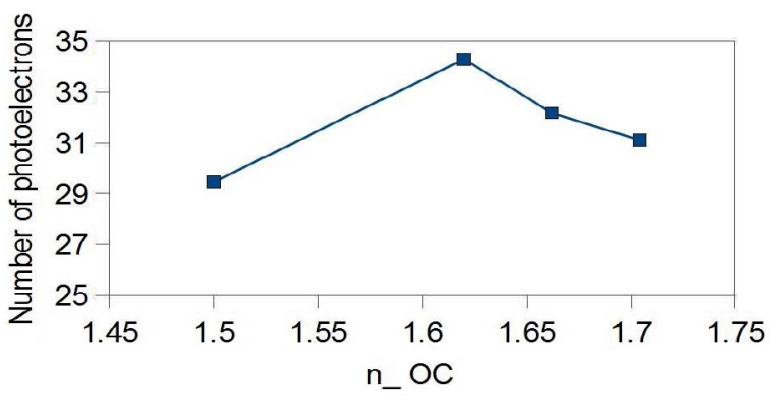

Fig. 5. Light detection dependence on optical coupling index $n_{-} \mathrm{OC}=$ 1.5/1.62/1.66/1.704. Scintillator thickness $H=1.27 \mathrm{~cm}$; surface roughness sigma $=3$; reflector foil reflectivity $\mathrm{RC}=0.98$.

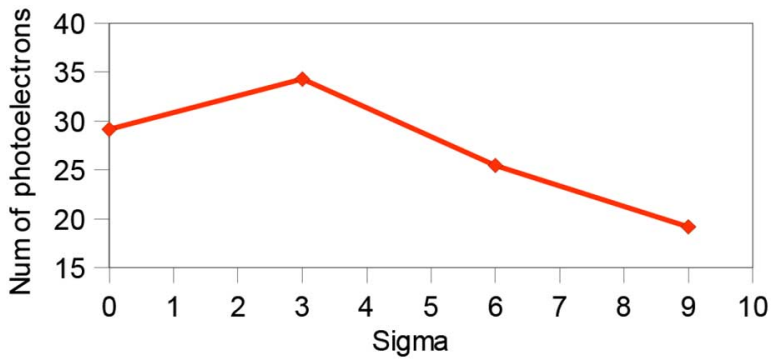

Fig. 6. Light detection dependence on scintillator surface roughness. Scintillator thickness $\mathrm{H}=2.5 \mathrm{~cm}$; optical coupling index n_OC $=1.62$; reflector foil reflectivity $\mathrm{RC}=0.98$.

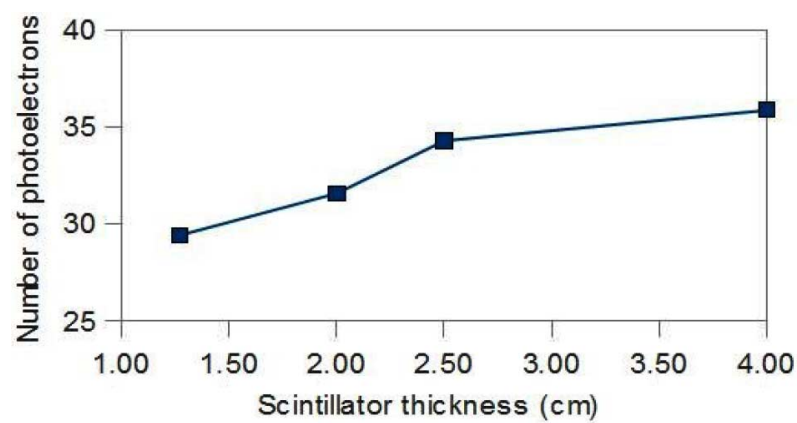

Fig. 7. PMT light signal dependence on scintillator thickness. Surface roughness sigma $=3$; optical coupling index $\mathrm{n}_{-} \mathrm{OC}=1.62$; reflector foil reflectivity $\mathrm{RC}=0.98$.

range of values $\mathrm{n}_{-} \mathrm{OC}=1.50$ to 1.70 . A good index matching can be obtained with $\mathrm{n}_{-} \mathrm{OC}=1.62$ (see Fig. 5).

Scintillator surface roughness: To study the influence of the scintillator surface roughness on light transport and collection, the roughness parameter is varied over a range of values sigma $=0$ to 9. From the results shown in Fig. 6 it is clear that scintillator surface roughness optimization to values of about sigma $=3.0$ has a major impact on the number of photons reaching the PMT area.

Scintillator thickness: To study the influence of the scintillator slab thickness on light production and collection, the thickness parameter is varied over a range of values $\mathrm{H}=1.27$ to $4.0 \mathrm{~cm}$. The results shown in Fig. 7 indicate that the use of thickness values greater than $2.5 \mathrm{~cm}$ has a low impact on the number of detected photons. This effect is explained by a decrease in the number of photons collected at the PMT when the scintillator thickness is large compared to the PMT window diameter,

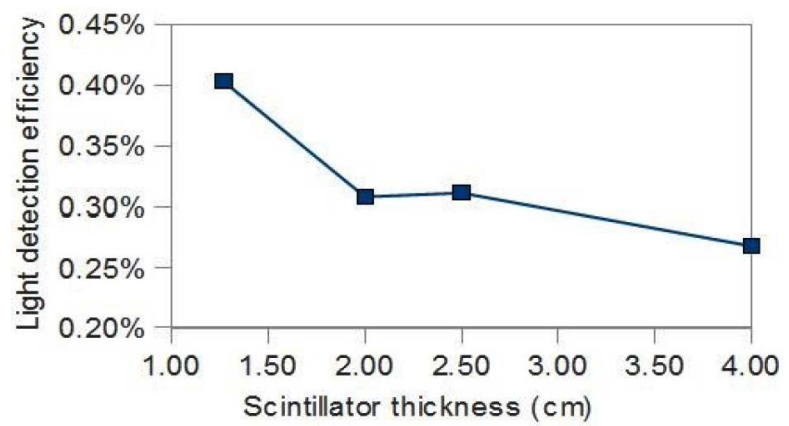

Fig. 8. Light detection efficiency (detected/emitted photons) dependence on scintillator thickness. Light collection decreases for scintillator thickness values very different to the PMT active area diameter. Surface roughness sigma $=3$; optical coupling index $\mathrm{n}_{-} \mathrm{OC}=1.62$; reflector foil reflectivity $\mathrm{RC}=0.98$.

TABLE II

Proposed Detector Module Configuration, Main Parameters Values OBTAINED FROM SIMULATIONS

\begin{tabular}{ll}
\hline Detector element & Simulation model parameter \\
\hline $\begin{array}{l}\text { Scintillator slab } \\
\text { (BC-408) }\end{array}$ & $\begin{array}{l}\text { Scintillator thickness: } \\
\mathrm{H}=2.5 \mathrm{~cm}\end{array}$ \\
$\begin{array}{l}\text { Scintillator surface finish } \\
\text { Polished slab }\end{array}$ & $\begin{array}{l}\text { Surface roughness: } \\
\text { sigma }=3.0\end{array}$ \\
Reflector foil & Reflector reflectivity: \\
& RC_reflec $=0.98$ \\
PMT photodetector & Optical coupling index: \\
Photocathode diameter: $2.5 \mathrm{~cm}$ & n_OC $=1.62$ \\
\hline
\end{tabular}

as shown in Fig. 8, where photon detection efficiency is defined as the ratio between the number of detected photons (photoelectrons) and the number of photons generated at the interaction point.

Proposed detector configuration: From the simulation results, one can identify detector parameter values (range) that maximize photon detection (and PMT light signal). The proposed values are summarized in Table II.

2) Photodetector light response function: The resulting PMT LRF obtained for the proposed detector module readout configuration is shown in Fig. 9. The mean number of detected photons decreases with the distance (from the PMT center) to the muon interaction point, following the distributions shown in Fig. 9. As expected, light signal values are proportional to the size of the PMT active area. The use of a $96 \%$ larger photodetector active area (3.5 $\mathrm{cm}$ vs $2.5 \mathrm{~cm}$ diameter) gives an average increase in photon detection of $88 \%$ on the LRF.

The LRF dispersion (shown in Fig. 10) is proportional to the statistical variations in light detection, and inversely proportional to the square root of the number of photoelectrons. An average improvement in LRF dispersion of $5 \%$ can be expected from $3.5 \mathrm{~cm}$ diameter active area PMTs.

3) Spatial Resolution: To evaluate the spatial resolution that can be obtained with the proposed detector module readout configuration using $3.5 \mathrm{~cm}$ diameter active area PMTs, a large number of muon interaction events (1000 events) have been simulated at two different points $(\mathrm{x}=0$ and $\mathrm{x}=6 \mathrm{~cm}$, $y=0$ ). The LSF represents the spatial distribution of the number of photons detected at the readout face by the PMTs. 


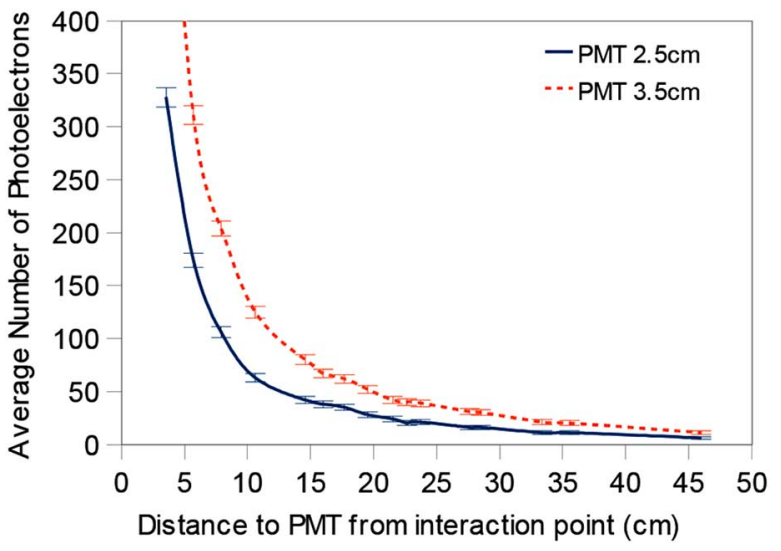

Fig. 9. Light signal dependence on distance to interaction point, PMT LRF, of the proposed detector module readout configuration $(2.5 \mathrm{~cm}$ scintillator thickness; surface roughness sigma $=3$; optical coupling index n_OC $=1.62$; reflector foil reflectivity $\mathrm{RC}=0.98$ ). Average light signal dependence on distance to the muon interaction point (light emission point). Results for two different photodetector sizes are shown: $2.5 \mathrm{~cm}$ diameter and $3.5 \mathrm{~cm}$ diameter active areas.

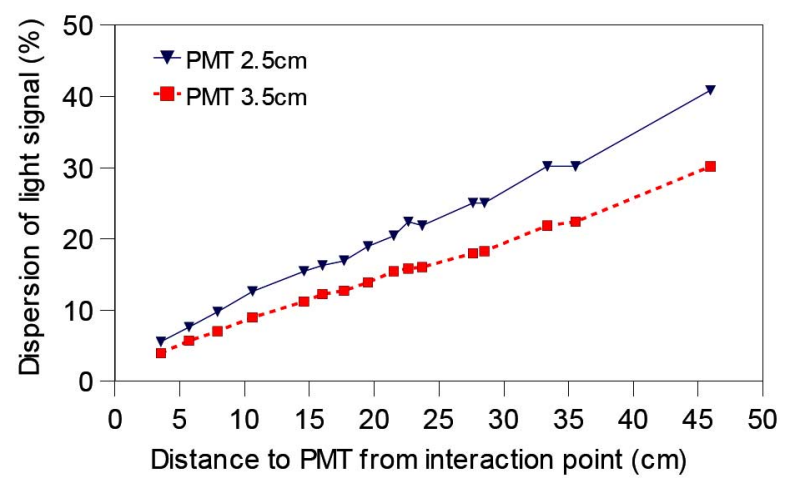

Fig. 10. Variation of light signal dispersion with distance to interaction point at the proposed readout configuration. LRF dispersion is clearly lower with $3.5 \mathrm{~cm}$ than with $2.5 \mathrm{~cm}$ diam PMT.
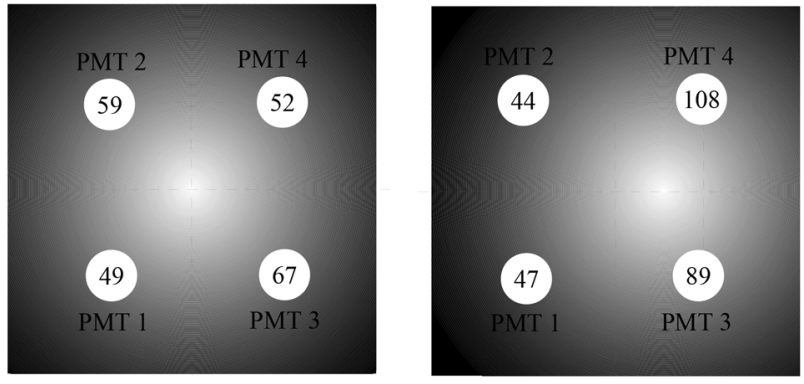

Fig. 11. Two different LSF set of values obtained at the $2 \times 2$ PMT array are shown, for a simulated muon interaction event at point $\mathrm{x}=0$ (left) and at point $\mathrm{x}=6 \mathrm{~cm}$ (right), on the $\mathrm{x}$-axis. Units are photoelectrons per event at a given PMT position. Although for interaction point $\mathrm{x}=0$ the four PMTs are equidistant to the light emission point, a different number of photoelectrons are obtained at each PMT due to statistical fluctuations in the light transmission and detection process. The shaded background represent the $(\mathrm{x}, \mathrm{y})$ distribution of photons reaching the slab surface.

For each event, the LSF is obtained as a 2D histogram of the photoelectrons collected in the $2 \times 2$ array of PMTs. Typical LSF values corresponding to muon interaction events at two separate interaction points are represented in Fig. 11.
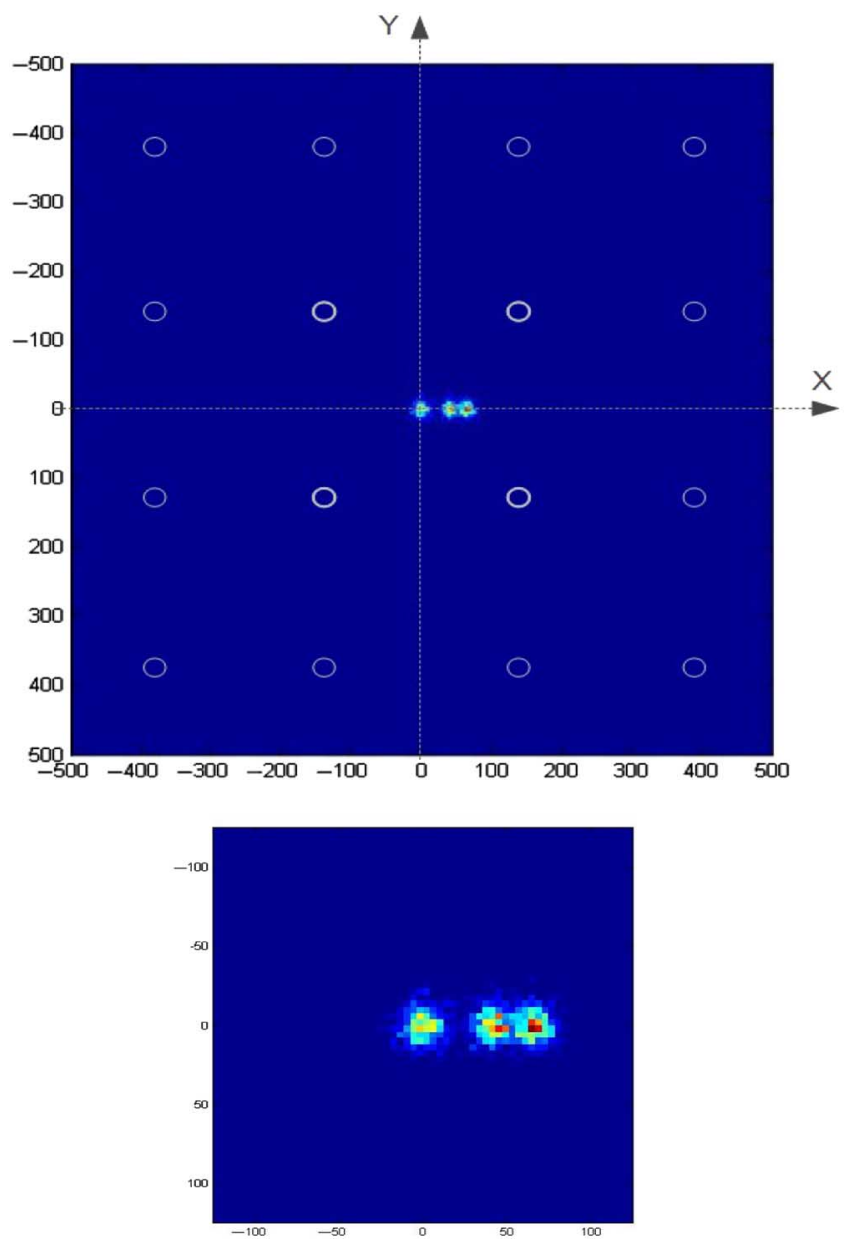

Fig. 12. Top: Planar image of the calculated point of interaction position distributions, PSF, corresponding to muon interactions at different points in the central region of the detector, $(\mathrm{x}, \mathrm{y})=(0,0),(60,0)$ and $(120,0)$. Units are in mm. Circles represent locations of PMTs. Only the 4 central PMTs are considered for the interaction position calculation. Bottom: Detail (zoom) of planar image central region showing well-resolved PSFs.

Distributions of the interaction point PSFs are obtained as a 2D histogram of the calculated interaction point coordinates, for all the muon interaction events simulated at two different points $(\mathrm{x}=0$ and $\mathrm{x}=6 \mathrm{~cm}, \mathrm{y}=0)$. Fig. 12 shows the resulting planar image of the calculated interaction point position distributions of muon interactions at the detector module, where well-resolved PSFs can be identified. The PSF profiles, obtained as a projection on the $\mathrm{x}$-axis of the PSFs and their Gaussian fits are shown in Fig. 13. The width of the resulting PSFs was estimated from Gaussian fits of the PSF profiles, and full width at half maximum (FWHM) values of $13 \mathrm{~mm}, 12 \mathrm{~mm}$ and $12 \mathrm{~mm}$ respectively was obtained. This result indicates that a detector intrinsic spatial resolution of $\sim 1-2 \mathrm{~cm}$ can be achieved at the center of the simulated detector module.

A degradation of the spatial resolution can be seen outside the central region. The PSF corresponding to muon interactions at $\mathrm{x}=6 \mathrm{~cm}$ and $\mathrm{x}=12 \mathrm{~cm}$ are centered at $\mathrm{x}=4 \mathrm{~cm}$ (bias $=-2 \mathrm{~cm}$ ) and $\mathrm{x}=6.5 \mathrm{~cm}$ (bias $=-5.5 \mathrm{~cm}$ ), respectively, showing a non-linear response (compression effect) of the centroid estimation algorithm. To take into account this bias for the estimation of the resolution, a first-order correction factor 


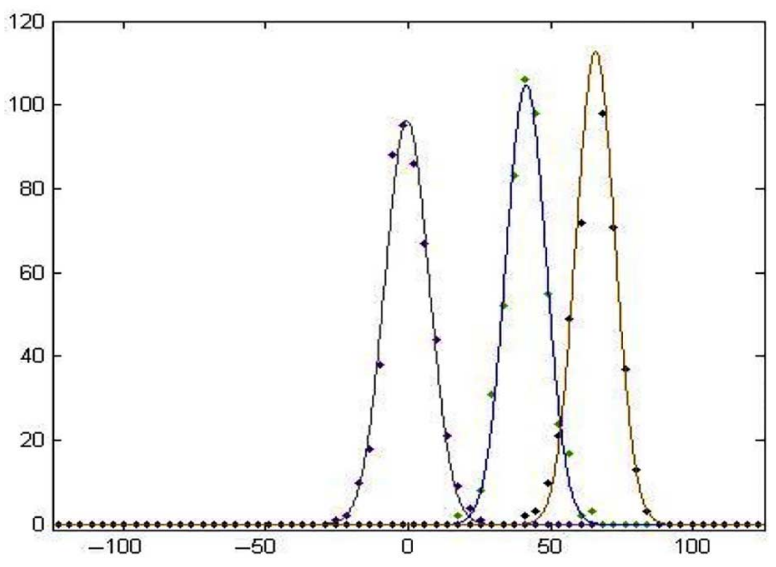

Fig. 13. Profile (projection on $\mathrm{x}$-axis) of PSFs corresponding to muon interactions at different points in the central region of the detector, $(x, y)=(0,0)$, $(60,0)$ and $(120,0)$. The resulting PSFs are centered at $\mathrm{x}=0, \mathrm{x}=40 \mathrm{~mm}$ and $\mathrm{x}=65 \mathrm{~mm}$, showing FWHM values of $13 \mathrm{~mm}, 12 \mathrm{~mm}$ and $12 \mathrm{~mm}$ respectively (Gaussian fits). X-coordinate units are in $\mathrm{mm}$.

on FWHM of interaction position/biased position can be considered, resulting in $\mathrm{FWHM}^{*} 6 / 4=18 \mathrm{~mm}$ at $\mathrm{x}=6 \mathrm{~cm}$ and $\mathrm{FWHM}^{*} 12 / 6.5=22 \mathrm{~mm}$ at $\mathrm{x}=12 \mathrm{~cm}$.

This is related to the truncation of the LSF when reaching the light detection acceptance limits of the $2 \times 2$ PMT readout region. A larger number of PMTs, as in a $4 \times 4$ PMT readout configuration, has to be considered for point of interaction calculation on a realistic detector module. Optimized detector response uniformity values are expected to be obtained by implementing statistical methods for interaction position determination, advanced processing methods as used in modern gamma cameras for Nuclear Medicine imaging (Maximum-Likelihood Estimation or Neural Networks).

These results are an initial estimation of the expected detector response, considering a simple square footprint distribution arrangement of small PMTs defined by a minimum spacing of $25 \mathrm{~cm}$. Other possible distributions could be considered (larger spacing, hexagonal footprint, etc.), but such a discussion would be part of a further optimization study, beyond the scope of this paper. After this basic detector model definition, a small prototype will be developed for the simulation model validation. These low-level results will be integrated into complex detection structure models for full muon radiography system simulation. We aim to develop a simulation tool for system performance evaluation (muon radiography spatial resolution and sensitivity) versus system cost (mainly determined by the number of photodetectors $/ \mathrm{m}^{2}$ ) prior to any muon radiography system construction.

\section{CONCLusions}

In our study we have addressed the possibility of using largearea square plastic scintillator slabs $(100 \mathrm{~cm} \times 100 \mathrm{~cm})$ as solid and robust detectors for muon radiography of large-volume objects. A simple arrangement of relatively small photodetectors (2.5-3.5 cm diameter active area PMTs) covering the scintillator surface has been considered, defining a square footprint sparse distribution, with $25 \mathrm{~cm}$ minimum spacing. A basic detector model has been defined using a Monte Carlo simulation toolkit GATE including optical transport. The feasibility of the proposed light readout scheme has been evaluated.

From simulation results, detector parameter values maximizing photon detection have been identified, like optimal scintillator surface roughness and thickness or PMT optical coupling. The light response function of PMTs $(3.5 \mathrm{~cm}$ diameter) for the proposed detector module readout configuration has been determined. A detector intrinsic spatial resolution range of 1.3-2.2 cm has been estimated from interaction point spread function distributions of muon interactions at the center region of the detector module.

\section{REFERENCES}

[1] E. P. George, "Cosmic rays measure overburden of tunnel," Commonwealth Engineer, pp. 455-457, Jul. 1, 1955.

[2] K. Nagamine et al., "Method of probing inner-structure of geophysical substance with the horizontal cosmic-ray muons and possible application to volcanic eruption prediction," Nucl. Instrum. Methods Phys. Res. A, vol. 356, p. 585, 1995.

[3] H. K. M. Tanaka et al., "High resolution imaging in the inhomogeneous crust with cosmic ray muon radiography: The density structure below the volcanic crater floor of Mt. Asama, Japan," Earth Planet. Sci. Lett. vol. 263, p. 104, 2007.

[4] G. Ambrosi et al., "The MU-RAY project: Volcano radiography with cosmic-ray muons," Nucl. Instrum. Methods Phys. Res. A, vol. 628, p. 120, 2011.

[5] S. Riggi et al., "Geant4 simulation of plastic scintillator strips with embedded optical fibers for a prototype of tomographic system," Nucl. Instrum. Methods Phys. Res. A, vol. 624, p. 583, 2010.

[6] K. N. Borozdin et al., "Radiographic Imaging with Cosmic Ray Muons," Nature, vol. 422, p. 277, 2003.

[7] M. Hohlmann et al., "GEANT4 simulation of a cosmic ray muon tomography system with micropattern gas detectors for the detection of HighZ materials," IEEE Trans. Nulc Sci., vol. 56, no. 3, pp. 1356-1363, Jun. 2009.

[8] S. Presente et al., "First results on material identification and imaging with a large-volume muon tomography prototype," Nucl. Instrum. Methods Phys. Res. A, vol. 604, p. 738, 2009.

[9] N. Lesparre et al., "Design and operation of a field telescope for cosmic ray geophysical tomography," Geosci. Instrum. Method. Data Syst., no. 1, pp. 33-42, 2012.

[10] S. Riggi et al., "Muon tomography imaging algorithms for nuclear threat detection inside large volume containers with the Muon Portal detector," Nucl. Instrum. Methods Phys. Res. A, vol. 728, p. 59, 2013.

[11] A. Clarkson et al., "The design and performance of a scintillating-fibre tracker for the cosmic-ray muon tomography of legacy nuclear waste containers," Nucl. Instrum. Methods Phys. Res. A, vol. 745, p. 138, 2014.

[12] [Online]. Available: www.opengatecollaboration.org

[13] [Online]. Available: www.detectors.saint-gobain.com

[14] P. Aguiar et al., "A feasibility study on the use of arrays of discrete SiPMs for MR compatible LYSO readout using Monte Carlo simulation," J. Instrum., vol. 7, p. P06002, 2012.

[15] Vikuiti Enhanced Specular Reflector Film (ESR) from 3M [Online]. Available: http://solutions.3m.com/wps/portal/3M/en_US/NA_Optical/

[16] H. O. Anger, "Scintillation camera," Rev. Sci. Instrum., vol. 29, p. 27, 1958. 